A N N A LES

UNIVERSITATIS MARIAE CURIE-SKŁODOWSKA

LUBLIN - POLONIA

\title{
Inequalities concerning the rate of growth of polynomials involving the polar derivative
}

\begin{abstract}
This paper contains some results for algebraic polynomials in the complex plane involving the polar derivative that are inspired by some classical results of Bernstein. Obtained results yield the polar derivative analogues of some inequalities giving estimates for the growth of derivative of lacunary polynomials.
\end{abstract}

1. Introduction. Let $P(z)=\sum_{v=0}^{n} a_{v} z^{v}$ be a polynomial of degree $n$ in the complex plane and $P^{\prime}(z)$ be its derivative. The Bernstein inequality that relates the norm of a polynomial to that of its derivative and its various versions form a classical topic in analysis. One basic result is that if $P(z)$ is a polynomial of degree $n$, then on $|z|=1$,

$$
\left|P^{\prime}(z)\right| \leq n \max _{|z|=1}|P(z)| \text {. }
$$

The inequality (1.1) is a famous result due to Bernstein [3], who proved it in 1912 and is best possible with the equality holding for polynomials $P(z)=\lambda z^{n}, \lambda$ being a complex number. Later, in 1930 (see [4]), Bernstein revisited his inequality (1.1) and established a more general result: for two polynomials $P(z)$ and $Q(z)$ with the degree of $P(z)$ not exceeding that of $Q(z)$ and $Q(z) \neq 0$ for $|z|>1$, the inequality $|P(z)| \leq|Q(z)|$ on the unit circle $|z|=1$ implies the inequality of their derivatives $\left|P^{\prime}(z)\right| \leq\left|Q^{\prime}(z)\right|$ on

2010 Mathematics Subject Classification. 30A10, 30C10, 30C15.

Key words and phrases. Bernstein inequality, lacunary polynomial, zeros. 
$|z|=1$. In fact, this inequality gives (1.1) in particular by taking $Q(z)=$ $z^{n} \max _{|z|=1}|P(z)|$.

These fundamental inequalities have been the starting point of a considerable literature on polynomial approximations and over a period, various versions and generalizations of these inequalities have been produced by introducing restrictions on the zeros, the modulus of largest root, restrictions on coefficients etc. If we restrict ourselves to the class of polynomials having no zero in $|z|<1$, then the inequality (1.1) can be sharpened. In fact, Erdös conjectured and later Lax [10] proved that if $P(z) \neq 0$ in $|z|<1$, then

$$
\max _{|z|=1}\left|P^{\prime}(z)\right| \leq \frac{n}{2} \max _{|z|=1}|P(z)| \text {. }
$$

As an extension of (1.2), Malik [11] proved that if $P(z) \neq 0$ in $|z|<k$, $k \geq 1$, then

$$
\max _{|z|=1}\left|P^{\prime}(z)\right| \leq \frac{n}{1+k} \max _{|z|=1}|P(z)| .
$$

Bidkham and Dewan [5] obtained a generalization of (1.3) and proved that if $P(z) \neq 0$ in $|z|<k, k \geq 1$, then for $1 \leq r \leq k$,

$$
\max _{|z|=r}\left|P^{\prime}(z)\right| \leq \frac{n(r+k)^{n-1}}{(1+k)^{n}} \max _{|z|=1}|P(z)| .
$$

By considering a more general class of polynomials $P(z)=a_{0}+\sum_{v=\mu}^{n} a_{v} z^{v}$, $1 \leq \mu \leq n$, not vanishing in $|z|<k, k \geq 1$, Aziz and Zargar [2] generalized (1.4) and obtained for $0<r \leq R \leq k$,

$$
\max _{|z|=R}\left|P^{\prime}(z)\right| \leq \frac{n R^{\mu-1}\left(k^{\mu}+R^{\mu}\right)^{\frac{n}{\mu}-1}}{\left(k^{\mu}+R^{\mu}\right)^{\frac{n}{\mu}}} \max _{|z|=r}|P(z)| .
$$

Further as an improvement of (1.5), Aziz and Shah [1] proved under the same hypothesis that

$$
\max _{|z|=R}\left|P^{\prime}(z)\right| \leq \frac{n R^{\mu-1}\left(k^{\mu}+R^{\mu}\right)^{\frac{n}{\mu}-1}}{\left(k^{\mu}+R^{\mu}\right)^{\frac{n}{\mu}}}\left\{\max _{|z|=r}|P(z)|-\min _{|z|=k}|P(z)|\right\},
$$

for $0<r \leq R \leq k$.

For a polynomial $P(z)$ of degree $n$, we define

$$
D_{\alpha} P(z):=n P(z)+(\alpha-z) P^{\prime}(z),
$$

the polar derivative of $P(z)$ with respect to the point $\alpha$. The polynomial $D_{\alpha} P(z)$ is of the degree at most $n-1$ and it generalizes the ordinary derivative in the following sense:

$$
\lim _{\alpha \rightarrow \infty}\left[\frac{D_{\alpha} P(z)}{\alpha}\right]=P^{\prime}(z)
$$

uniformly with respect to $z$ for $|z| \leq R, R>0$. 
Over the last four decades many different authors produced a large number of results and established various Bernstein type inequalities for polynomials involving the polar derivative $D_{\alpha} P(z)$ with various choices of $P(z)$, $\alpha$ and other parameters. The obtained inequalities generalize some known results for ordinary derivatives and provide crucial tools in obtaining inverse theorems in approximation theory. For the latest research and development on this topic one can consult the papers $[7,12-15,17]$. Recently, Somsuwan and Nakprasit [17] extended (1.6) for the case $k \geq 1$ to the polar derivative of $P(z)$ and proved the following result.

Theorem A. If $P(z)=a_{0}+\sum_{v=\mu}^{n} a_{v} z^{v}, 1 \leq \mu \leq n$, is a polynomial of degree $n$ having no zeros in $|z|<k, k \geq 1$, then for every $\alpha \in \mathbb{C}$ with $|\alpha| \geq R$ and $0<r \leq R \leq k$,

$$
\max _{|z|=R}\left|D_{\alpha} P(z)\right| \leq \frac{n}{1+s_{0}}\left[\max _{|z|=r}|P(z)| E_{0}-\left\{E_{0}-\left(s_{0}+1\right)\right\} m\right],
$$

where

$$
\begin{aligned}
E_{0} & =\left(\frac{|\alpha|}{R}+s_{0}\right)\left(\frac{k^{\mu}+R^{\mu}}{k^{\mu}+r^{\mu}}\right)^{\frac{n}{\mu}}, \\
s_{0} & =\left(\frac{k}{R}\right)^{\mu+1}\left\{\frac{\left(\frac{\mu}{n}\right) \frac{\left|a_{\mu}\right|}{\left|a_{0}\right|-m} R k^{\mu-1}+1}{\left(\frac{\mu}{n}\right) \frac{\left|a_{\mu}\right|}{R\left(\left|a_{0}\right|-m\right)} k^{\mu+1}+1}\right\}
\end{aligned}
$$

and $m=\min _{|z|=k}|P(z)|$.

2. Main Results. The main aim of this paper is to prove a more general result for the polar derivative of a polynomial which not only provides a refinement and extension of Theorem A, but as a special case strengthens inequality (1.6) as well. Our result and its consequences also give the polar derivative analogues of some estimates for the growth of derivative of lacunary polynomial.

Theorem 1. If $P(z)=a_{0}+\sum_{v=\mu}^{n} a_{v} z^{v}, 1 \leq \mu \leq n$, is a polynomial of degree $n$ having no zeros in $|z|<k, k>0$, then for $0<r \leq R \leq k$, $0 \leq t \leq 1$ and for every $\alpha \in \mathbb{C}$ with $|\alpha| \geq R$,

$$
\max _{|z|=R}\left|D_{\alpha} P(z)\right| \leq \frac{n}{1+s_{t}}\left[\max _{|z|=r}|P(z)| E_{t}-\left\{E_{t}-\left(s_{t}+1\right)\right\} t m\right],
$$

where

$$
\begin{aligned}
E_{t} & =\left(\frac{|\alpha|}{R}+s_{t}\right) \exp \left(n \int_{r}^{R} A_{\varsigma} d \varsigma\right), \\
s_{t} & =\left(\frac{k}{R}\right)^{\mu+1}\left\{\frac{\left(\frac{\mu}{n}\right) \frac{\left|a_{\mu}\right|}{\left|a_{0}\right|-t m} R k^{\mu-1}+1}{\left(\frac{\mu}{n}\right) \frac{\left|a_{\mu}\right|}{R\left(\left|a_{0}\right|-t m\right)} k^{\mu+1}+1}\right\},
\end{aligned}
$$




$$
A_{\varsigma}=\left\{\frac{\left(\frac{\mu}{n}\right) \frac{\left|a_{\mu}\right|}{\left|a_{0}\right|-t m} \varsigma^{\mu-1} k^{\mu+1}+\varsigma^{\mu}}{\varsigma^{\mu+1}+\left(\frac{\mu}{n}\right) \frac{\left|a_{\mu}\right|}{\left|a_{0}\right|-t m}\left(k^{\mu+1} \varsigma^{\mu}+k^{2 \mu} \varsigma\right)+k^{\mu+1}}\right\}
$$

and $m=\min _{|z|=k}|P(z)|$.

Remark 1. Since $P(z) \neq 0$ in $|z|<k, k>0$, the polynomial $T(z)=$ $P(\varsigma z) \neq 0$ in $|z|<\frac{k}{\varsigma}, \frac{k}{\varsigma} \geq 1$, where $0<\varsigma \leq k$. Hence applying inequality (3.3) of Lemma 1 to $T(z)$, we get for $0 \leq t \leq 1$,

$$
\frac{\left|a_{\mu}\right| \varsigma^{\mu}}{\left|a_{0}\right|-t m}\left(\frac{k}{\varsigma}\right)^{\mu} \leq \frac{n}{\mu}
$$

where

$$
m=\min _{|z|=\frac{k}{\varsigma}}|T(z)|=\min _{|z|=\frac{k}{\varsigma}}|P(\varsigma z)|=\min _{|z|=k}|P(z)| .
$$

Now, (2.2) becomes

$$
\left(\frac{\mu}{n}\right) \frac{\left|a_{\mu}\right| k^{\mu}}{\left|a_{0}\right|-t m} \leq 1
$$

which is equivalent to

$$
\frac{\left(\frac{\mu}{n}\right) \frac{\left|a_{\mu}\right|}{\left|a_{0}\right|-t m} \varsigma^{\mu-1} k^{\mu+1}+\varsigma^{\mu}}{\varsigma^{\mu+1}+\left(\frac{\mu}{n}\right) \frac{\left|a_{\mu}\right|}{\left|a_{0}\right|-t m}\left(k^{\mu+1} \varsigma^{\mu}+k^{2 \mu} \varsigma\right)+k^{\mu+1}} \leq \frac{\varsigma^{\mu-1}}{\varsigma^{\mu}+k^{\mu}} .
$$

Integrating both sides of (2.3) with respect to $\varsigma$ from $r$ to $R$, where $0<r \leq$ $R \leq k$, we get

$$
n \int_{r}^{R} \frac{\left(\frac{\mu}{n}\right) \frac{\left|a_{\mu}\right|}{\left|a_{0}\right|-t m} \varsigma^{\mu-1} k^{\mu+1}+\varsigma^{\mu}}{\varsigma^{\mu+1}+\left(\frac{\mu}{n}\right) \frac{\left|a_{\mu}\right|}{\left|a_{0}\right|-t m}\left(k^{\mu+1} \varsigma^{\mu}+k^{2 \mu} \varsigma\right)+k^{\mu+1}} d \varsigma \leq n \int_{r}^{R} \frac{\varsigma^{\mu-1}}{\varsigma^{\mu}+k^{\mu}} d \varsigma,
$$

which is equivalent to

$$
\exp \left\{n \int_{r}^{R} A_{\varsigma} d \varsigma\right\} \leq\left(\frac{k^{\mu}+R^{\mu}}{k^{\mu}+r^{\mu}}\right)^{\frac{n}{\mu}}
$$

Remark 2. Note that for every $r \leq k$, by Lemma 5 , the inequality

$$
\max _{|z|=r}|P(z)| \geq t m
$$

holds for every $t$ with $0 \leq t \leq 1$ and hence the function

$$
\frac{n}{1+s_{t}}\left[x \max _{|z|=r}|P(z)|-\left\{x-\left(s_{t}+1\right)\right\} t m\right]
$$

is a non-decreasing function of $x$. If we combine this fact with inequality (2.4), we get the following generalization and extension of Theorem A. 
Corollary 1. If $P(z)=a_{0}+\sum_{v=\mu}^{n} a_{v} z^{v}, 1 \leq \mu \leq n$, is a polynomial of degree $n$ having no zeros in $|z|<k, k>0$, then for $0<r \leq R \leq k$, $0 \leq t \leq 1$ and for every $\alpha \in \mathbb{C}$ with $|\alpha| \geq R$,

$$
\max _{|z|=R}\left|D_{\alpha} P(z)\right| \leq \frac{n}{1+s_{t}}\left[E_{t}^{*} \max _{|z|=r}|P(z)|-\left\{E_{t}^{*}-\left(s_{t}+1\right)\right\} t m\right],
$$

where $E_{t}^{*}=\left(\frac{|\alpha|}{R}+s_{t}\right)\left(\frac{k^{\mu}+R^{\mu}}{k^{\mu}+r^{\mu}}\right)^{\frac{n}{\mu}}, m=\min _{|z|=k}|P(z)|$ and $s_{t}$ is as defined in Theorem 1.

Dividing both sides of (2.5) by $|\alpha|$ and letting $|\alpha| \rightarrow \infty$, we get the following result.

Corollary 2. If $P(z)=a_{0}+\sum_{v=\mu}^{n} a_{v} z^{v}, 1 \leq \mu \leq n$, is a polynomial of degree $n$ having no zeros in $|z|<k, k>0$, then for $0<r \leq R \leq k$ and $0 \leq t \leq 1$, we get

$$
\max _{|z|=R}\left|P^{\prime}(z)\right| \leq \frac{n}{R\left(1+s_{t}\right)}\left(\frac{k^{\mu}+R^{\mu}}{k^{\mu}+r^{\mu}}\right)^{\frac{n}{\mu}}\left\{\max _{|z|=r}|P(z)|-t m\right\},
$$

where $m=\min _{|z|=k}|P(z)|$ and $s_{t}$ is as defined in Theorem 1 .

Remark 3. It is easy to see that

$$
\frac{1}{R\left(1+s_{t}\right)}=\frac{\left(\frac{\mu}{n}\right) \frac{\left|a_{\mu}\right|}{\left|a_{0}\right|-t m} R^{\mu-1} k^{\mu+1}+R^{\mu}}{R^{\mu+1}+\left(\frac{\mu}{n}\right) \frac{\left|a_{\mu}\right|}{\left|a_{0}\right|-t m}\left(k^{\mu+1} R^{\mu}+k^{2 \mu} R\right)+k^{\mu+1}},
$$

and since $R \leq k$, if we put $\varsigma=R$ in (2.3), we get

$$
\frac{1}{R\left(1+s_{t}\right)} \leq \frac{R^{\mu-1}}{R^{\mu}+k^{\mu}}
$$

If we use the inequality (2.6) in Corollary 2, we get the following generalization of (1.6).

Corollary 3. If $P(z)=a_{0}+\sum_{v=\mu}^{n} a_{v} z^{v}, 1 \leq \mu \leq n$, is a polynomial of degree $n$ having no zeros in $|z|<k, k>0$, then for $0<r \leq R \leq k$ and $0 \leq t \leq 1$, we get

$$
\max _{|z|=R}\left|P^{\prime}(z)\right| \leq \frac{n R^{\mu-1}\left(k^{\mu}+R^{\mu}\right)^{\frac{n}{\mu}-1}}{\left(k^{\mu}+R^{\mu}\right)^{\frac{n}{\mu}}}\left\{\max _{|z|=r}|P(z)|-t m\right\},
$$

where $m=\min _{|z|=k}|P(z)|$. Equality in (2.7) holds for $P(z)=\left(z^{\mu}+k^{\mu}\right)^{\frac{n}{\mu}}$, where $n$ is a multiple of $\mu$.

Remark 4. Many other interesting results can be deduced from the above results for different choices of the parameters. For example, if we divide both sides of inequality (2.1) of Theorem 1 by $|\alpha|$, let $|\alpha| \rightarrow \infty$ and take $t=1$, we get a result of Chanam and Dewan ([6], Theorem 2) and for 
$r=R=1$ and $t=1$, it implies a result of Dewan, Singh and Mir [7]. Further for $t=1$, Corollary 3 reduces to (1.6).

3. Lemmas. We need the following lemmas to prove Theorem 1.

Lemma 1. If $P(z)=a_{0}+\sum_{v=\mu}^{n} a_{v} z^{v}, 1 \leq \mu \leq n$, is a polynomial of degree $n$ having no zeros in $|z|<k, k \geq 1$, then for $0 \leq t \leq 1$ and $|z|=1$,

$$
\left|P^{\prime}(z)\right| \leq \frac{n}{1+B_{t}}\left\{\max _{|z|=1}|P(z)|-t m\right\}
$$

where

$$
\begin{gathered}
B_{t}=k^{\mu+1}\left\{\frac{\left(\frac{\mu}{n}\right) \frac{\left|a_{\mu}\right|}{\left|a_{0}\right|-t m} k^{\mu-1}+1}{\left(\frac{\mu}{n}\right) \frac{\left|a_{\mu}\right|}{\left|a_{0}\right|-t m} k^{\mu+1}+1}\right\}, \\
\left(\frac{\mu}{n}\right) \frac{\left|a_{\mu}\right| k^{\mu}}{\left|a_{0}\right|-t m} \leq 1
\end{gathered}
$$

and $m=\min _{|z|=k}|P(z)|$.

Lemma 2. If $P(z)=a_{0}+\sum_{v=\mu}^{n} a_{v} z^{v}, 1 \leq \mu \leq n$, is a polynomial of degree $n$ having no zeros in $|z|<k, k>0$, then for $0<r \leq R \leq k$ and $0 \leq t \leq 1$,

$$
\max _{|z|=R}|P(z)| \leq \exp \left(n \int_{r}^{R} A_{\varsigma} d \varsigma\right) \max _{|z|=r}|P(z)|+\left\{1-\exp \left(n \int_{r}^{R} A_{\varsigma} d \varsigma\right)\right\} t m,
$$

where $m=\min _{|z|=k}|P(z)|$ and $A_{\varsigma}$ is as defined in Theorem 1 .

The above two lemmas are due to Mir and Dar [16].

Lemma 3. If $P(z)$ is a polynomial of degree $n$, then on $|z|=1$, we have

$$
\left|P^{\prime}(z)\right|+\left|Q^{\prime}(z)\right| \leq n \max _{|z|=1}|P(z)|,
$$

where $Q(z)=z^{n} \overline{P\left(\frac{1}{\bar{z}}\right)}$.

The above lemma is a special case of a result due to Govil and Rahman [9].

Lemma 4. If $P(z)=a_{0}+\sum_{v=\mu}^{n} a_{v} z^{v}, 1 \leq \mu \leq n$, is a polynomial of degree $n$ having no zeros in $|z|<k, k \geq 1$, then for every $\alpha \in \mathbb{C}$ with $|\alpha| \geq 1$, and $0 \leq t \leq 1$,

$$
\max _{|z|=1}\left|D_{\alpha} P(z)\right| \leq \frac{n}{1+B_{t}}\left\{\left(|\alpha|+B_{t}\right) \max _{|z|=1}|P(z)|-(|\alpha|-1) t m\right\},
$$

where $m=\min _{|z|=k}|P(z)|$ and $B_{t}$ is defined by formula (3.2). 
Proof of Lemma 4. If $Q(z)=z^{n} \overline{P\left(\frac{1}{\bar{z}}\right)}$, then it is easy to verify for $|z|=1$, that

$$
\left|Q^{\prime}(z)\right|=\left|n P(z)-z P^{\prime}(z)\right| .
$$

Also for every $\alpha \in \mathbb{C}$ with $|\alpha| \geq 1$, we have

$$
D_{\alpha} P(z)=n P(z)+(\alpha-z) P^{\prime}(z) .
$$

Using this formula, equality (3.5) and Lemma 3 for $|z|=1$ and $|\alpha| \geq 1$, we obtain

$$
\begin{aligned}
\left|D_{\alpha} P(z)\right| & \leq\left|n P(z)-z P^{\prime}(z)\right|+|\alpha||| P^{\prime}(z) \mid \\
& =\left|Q^{\prime}(z)\right|+|\alpha|\left|P^{\prime}(z)\right| \\
& =\left|Q^{\prime}(z)\right|+\left|P^{\prime}(z)\right|-\left|P^{\prime}(z)\right|+|\alpha|\left|P^{\prime}(z)\right| \\
& \leq n \max _{|z|=1}|P(z)|+(|\alpha|-1)\left|P^{\prime}(z)\right| .
\end{aligned}
$$

Inequality (3.6) in conjunction with (3.1) of Lemma 1 gives for $|z|=1$ and $|\alpha| \geq 1$,

$$
\left|D_{\alpha} P(z)\right| \leq n \max _{|z|=1}|P(z)|+(|\alpha|-1)\left\{\frac{n}{1+B_{t}}\left(\max _{|z|=1}|P(z)|-t m\right)\right\},
$$

from which we can obtain (3.4).

The following lemma is due to Gardner, Govil and Musukula [8].

Lemma 5. If $P(z)=\sum_{v=0}^{n} a_{v} z^{v}$ is a polynomial of degree $n$ having no zeros in $|z|<k, k>0$, then $|P(z)| \leq m$ for $|z| \leq k$, where $m=\min _{|z|=k}|P(z)|$.

4. Proof of Theorem 1. Recall that $P(z) \neq 0$ in $|z|<k, k>0$, the polynomial $T(z)=P(R z)$, where $0<R \leq k$, has no zero in $|z|<\frac{k}{R}$, $\frac{k}{R} \geq 1$. Now applying inequality (3.4) of Lemma 4 to the polynomial $T(z)$ and noting that $|\alpha| \geq R$, we get

$$
\begin{aligned}
\max _{|z|=1} \mid & D_{\frac{\alpha}{R}} T(z) \mid \\
& \leq \frac{n}{1+s_{t}}\left\{\left(\frac{|\alpha|}{R}+s_{t}\right) \max _{|z|=1}|T(z)|-\left(\frac{|\alpha|}{R}-1\right) t \min _{|z|=\frac{k}{R}}|T(z)|\right\},
\end{aligned}
$$

where $s_{t}$ is defined in Theorem 1 .

We apply the relations

$$
\max _{|z|=1}\left|D_{\frac{\alpha}{R}} T(z)\right|=\max _{|z|=R}\left|D_{\alpha} P(z)\right|, \max _{|z|=1}|T(z)|=\max _{|z|=R}|P(z)|
$$

and

$$
\min _{|z|=\frac{k}{R}}|T(z)|=\min _{|z|=k}|P(z)|=m
$$


into (4.1) and obtain

(4.2) $\max _{|z|=R}\left|D_{\alpha} P(z)\right| \leq \frac{n}{1+s_{t}}\left\{\left(\frac{|\alpha|}{R}+s_{t}\right) \max _{|z|=R}|P(z)|-\left(\frac{|\alpha|}{R}-1\right) t m\right\}$.

From Lemma 2, we have for $0<r \leq R \leq k$ and $0 \leq t \leq 1$,

$$
\begin{aligned}
\max _{|z|=R}|P(z)| \leq & \exp \left(n \int_{r}^{R} A_{\varsigma} d \varsigma\right) \max _{|z|=r}|P(z)| \\
& +\left\{1-\exp \left(n \int_{r}^{R} A_{\varsigma} d \varsigma\right)\right\} t m,
\end{aligned}
$$

where $A_{\varsigma}$ is defined in Theorem 1. Using (4.3) in (4.2), we obtain

$$
\max _{|z|=R}\left|D_{\alpha} P(z)\right| \leq \frac{n}{1+s_{t}}\left[\max _{|z|=r}|P(z)| E_{t}-\left\{E_{t}-\left(s_{t}+1\right)\right\} t m\right],
$$

where $E_{t}$ is defined in Theorem 1 . This proves Theorem 1 completely.

Acknowledgements. The authors are very grateful to the reviewer for his/her comments that greatly improved the manuscript.

\section{REFERENCES}

[1] Aziz, A., Shah, W. M., Inequalities for a polynomial and its derivative, Math. Inequal. Appl. 7 (2004), 379-391.

[2] Aziz, A., Zargar, B. A., Inequalities for a polynomial and its derivative, Math. Inequal. Appl. 1 (1998), 543-550.

[3] Bernstein, S., Sur l'ordre de la meilleure approximation des functions continues par des polynômes de degré donné, Mem. Acad. R. Belg. 4 (1912), 1-103.

[4] Bernstein, S., Sur la limitation des derivées des polynômes, C. R. Acad. Sci. Paris 190 (1930), 338-341.

[5] Bidkham, M., Dewan, K. K., Inequalities for a polynomial and its derivative, J. Math. Anal. Appl., 166 (1992), 319-324.

[6] Chanam, B., Dewan, K. K., Inequalities for a polynomial and its derivative, J. Math. Anal. Appl. 336 (2007), 171-179.

[7] Dewan, K. K., Singh, N., Mir, A., Extensions of some polynomial inequalities to the polar derivative, J. Math. Anal. Appl. 352 (2009), 807-815.

[8] Gardner, R. B., Govil, N. K., Musukula, S. R., Rate of growth of polynomials not vanishing inside a circle, J. Inequal. Pure Appl. Math. 6 (2), Art. 53, (2005), 1-9.

[9] Govil, N. K., Rahman, Q. I., Functions of exponential type not vanishing in a half plane and related polynomials, Trans. Amer. Math. Soc. 137 (1969), 501-517.

[10] Lax, P. D., Proof of a conjecture of P. Erdös on the derivative of a polynomial, Bull. Amer. Math. Soc. 50 (1944), 509-513.

[11] Malik, M. A., On the derivative of a polynomial, J. London. Math. Soc. 1 (1969), 57-60.

[12] Mir, A., Hussain, I., On the Erdös-Lax inequality concerning polynomials, C. R. Acad. Sci. Paris, Ser. I 355 (2017), 1055-1062.

[13] Mir, A., On an operator preserving inequalities between polynomials, Ukrainian Math. J. 69 (2018), 1234-1247. 
[14] Mir, A., Dar, B., On the polar derivative of a polynomial, J. Ramanujan Math. Soc., 29 (2014), 403-412.

[15] Mir, A., Wani, A., Polynomials with polar derivatives, Funct. Approx. Comment. Math. 55 (2016), 139-144.

[16] Mir, A., Dar, B., Inequalities concerning the rate of growth of polynomials, Afr. Mat. 27 (2016), 279-290.

[17] Somsuwan, J., Nakprasit, K. N., Some bounds for the polar derivative of a polynomial, Int. J. Math. Math. Sci. (2018), Art. ID 5034607, 4 pp.

Abdullah Mir

Department of Mathematics

University of Kashmir

Srinagar, 190006

India

e-mail: drabmir@yahoo.com

Received June 24, 2019
Adil Hussain Malik

Department of Mathematics

University of Kashmir

Srinagar, 190006

India

e-mail: malikadil6909@gmail.com 